\title{
Application of LORA Spread Spectrum Technology On Intelligent Water Meter
}

\author{
Wenchang $\mathrm{Wu}^{1}$, Weida Sun ${ }^{2}$ \\ ${ }^{1}$ School of Electron and Information Engineering, Ningbo University of Technology, Ningbo, 315211 \\ 2.Ningbo Mingrui Sensors CO.,LTD., Ningbo, 315104
}

Keywords: LORA Spread Spectrum, Intelligent Water Meter, Application Research

\begin{abstract}
Spread spectrum technology is a new technology to make full use of limited spectrum resources and improve the efficiency of wireless information transmission. This paper introduces the working principle and system model of spread spectrum communication, and elaborates two main technologies of spread spectrum communication: Direct Sequence Spread Spectrum And frequency hopping spread spectrum, explain its working principle, and compare the performance of the two. In addition, the combination of spread spectrum and digital watermarking technology is introduced, and digital watermarking technology with spread spectrum is very robust and safe.
\end{abstract}

\section{Introduction}

With the wireless communications industry, the development of technology, 2013, the use of LORA spread spectrum technology, wireless communications products have begun to promote the field in the field of civilian meter reading, spread spectrum wireless table in the tap water industry applications in the initial stage, the majority of domestic water companies do not have large quantities Install the application. In order to better understand the application of spread spectrum technology in the actual environment, to provide a reference for the development of the sub-spread spectrum table technical standards, in 2015, Zhengzhou Water Company early narrowband wireless table as a whole to reduce the low rate of two residential quarters Pilot Application of Spread Spectrum Wireless Table. In this paper, through the application of spread spectrum wireless meter reading system, this paper introduces the characteristics of spread spectrum wireless meter reading technology, analyzes the application of wireless meter reading in pilot area, and puts forward some suggestions from the management and technical point of view.

\section{LORA Spread Spectrum Technology}

2013 Semtech Corporation introduced based on $1 \mathrm{GHz}$ below the ultra-long-distance low-power data transmission technology (LORA) chip, LORA is a dedicated radio frequency modulation and demodulation technology, and other such as FSK (frequency shift keying ), GMSK (Gaussian minimum frequency shift keying), BPSK (binary phase shift keying) and its derived modulation scheme to form a clear contrast.

2013 Semtech Corporation introduced based on 1GHz below the ultra-long-distance low-power data transmission technology (LORA) chip, LORA is a dedicated radio frequency modulation and demodulation technology, and other such as FSK (frequency shift keying ), GMSK (Gaussian minimum frequency shift keying), BPSK (binary phase shift keying) and its derived modulation scheme to form a clear contrast. It combines digital spread spectrum, digital signal processing and forward error correction coding technology, with unprecedented performance, to low transmit power to obtain a wider transmission range and distance. Previously, only some of the military communications system will be the integration of these technologies, and with the introduction of LORA technology, embedded wireless communications situation has undergone a radical change.

\section{Analysis of Water Meter Reading Table}

At present, the water meter widely used meter reading mainly prepaid IC card and smart meter 
remote meter reading system.

Prepaid IC card table fundamentally changed the consumption of people after the first settlement of consumer ideas for the industry authorities in advance to return funds and malicious fees for users to provide an effective means of management, but with the practical application of IC cards, more and more The more problems are emerging. At present, the main problem of prepaid IC card table is reflected in the following two aspects: prepaid IC card table need IC card as a recharging carrier, and the current IC card security there is a certain risk, commonly used IC card is decrypted or cracked Situation, to the water company to bring business risks. "The prepaid IC card table has no remote data transmission route, the water company can not timely copy the gas consumption and the usage statistics, can not accurately calculate the output difference and financial analysis.At the same time, IC card table malicious hoarding gas consumption, can not solve the price adjustment; Can not monitor the user's use of the drawbacks.

Based on the various drawbacks of prepaid IC card table, in order to effectively solve the timely and accurate data collection and prepaid and ladder pricing requirements, smart meter remote meter reading system as the best solution, its products have developed rapidly and become smart table future development trend. From the technical principle to analyze the remote table products are mainly two core technologies: reading acquisition technology and data transmission technology.

Reading acquisition technology that is how to ordinary mechanical film readings into electronic reading; the current smart table mainly uses two mainstream technologies, two technologies are more mature, each has its own advantages and disadvantages. The Pulse counting technology. The pulse count is a pulse sensor mounted on the counter turning part of the meter and accumulates and converts the resulting pulse signal to produce an electronic reading. The advantages of this counting technology is simple "low cost, easy to produce." The disadvantage is to 24 hours power supply, the pulse sensor to be 24 hours power supply, can not be collected and accumulated pulse signal; in the backup power shortage or power The case of pulse data is completely lost, resulting in meter reading and mechanical readings are inconsistent, need to be back after the call to be re-school table, easy and the user disputes. In addition, this counting technology is also prone to secondary cumulative error, Various disturbances (such as strong magnetic attack) or magnetic steel reed performance degradation, easy to cause more or less pulse.

Photoelectric direct reading counting technology. Photoelectric direct reading is installed in the table with a photoelectric character wheel position sensor, each word as a wheel, measure the absolute angle of the character wheel position, direct identification of the table with the window of the word number, so as to accurately obtain the table with a reading, There is no need for quadratic cumulative conversion. The method of table-side electronic reading device only in the meter when the power, copy power outage, low power consumption, there is no cumulative error, no power outage "external magnetic field interference, meter reading accuracy of $100 \%$. This counting technology The advantage is that no 24 hours without interruption of power supply, just meter meter instantaneous power supply to complete the reading conversion; power or undervoltage will not produce loss of reading. Reading accuracy is high, no secondary cumulative error; anti-interference ability, Or the electric pulse has no effect on it.Price is that this kind of counting technology structure is complex, the cost is higher.

Data transmission technology mainly refers to how the electronic reading effectively and reliably remote transmission to the water company management center. There are three main transmission technology solutions: cable transmission "short-range micro-power wireless transmission and mobile communication network transmission.

Wired transmission is the use of M-BUS bus transmission mode, the data transmission M-BUS bus with no two-wire system, power supply and data transmission are completed in two lines. The advantage is the use of optical direct reading technology, reading conversion accuracy is high; late transmission stability, copy table success rate is high. The shortcomings of the construction work is large, pre-decoration damage more serious. 


\section{Intelligent water meter system based on LORA spread spectrum technology}

Spread spectrum wireless meter reading system consists of the company master station management system, the district concentrator and the handset, the client spread spectrum table three levels structure. The main station and the concentrator through the GPRS public network for remote command and data bi-directional transmission, concentrator and table form a star network to point to point direct meter reading communication, the final realization of the terminal meter with remote meter reading data upload and valve control management. At the same time, the user site can also use the handheld to extract concentrator storage data or initiate real-time meter reading work on the table.

Spread spectrum wireless table to provide active and passive two wake-up meter reading model to meet the water company's different business needs. Two modes can be set up independent wireless communication parameters, including modulation, center frequency, transmission rate, spread spectrum factor. The active reporting mode is to automatically send the current data by automatically raising the frequency and the time at which the table automatically sends up the current data by collecting the current data by the concentrator. The passive receiving mode is real-time through the concentrator or the handset Sexual awake meter reading mode. As the spread spectrum wireless communication distance is relatively far, if the passive mode of the user-initiated group of meter reading, meter reading process will be on the signal coverage within the table with a frequent wake up, virtually increase the table with a battery consumption, and active Mode is omitted from the external wake-up process, can automatically wake up to complete the data meter reading upload work, so the pilot selection of wireless table active mode for daily automatic meter reading data billing, passive mode for a small number of unused table supplement, remote Real-time control and fault maintenance links.

After the system is put into operation, the company organizes hand to the scene with the handheld machine to read the table directly, as of November 2015 for a total of 10 on-site meter reading, a copy of the success rate of $98 \%$ or more, part of the household check Users to read the data comparison accuracy rate of $100 \%$. Master to the concentrator, concentrator to the end of the two-stage network uplink, downlink channel reliability is to ensure automatic meter reading and valve control, and prepaid business basis. Active mode, select part of the table to take a daily zero time to upload data, the concentrator sent to the master system, verify the automatic meter reading data upload success rate, as of December 2015, once a day to read the data to the master station success Rate of $96 \%$. Passive mode are from the main station real-time remote control valve remote control valve operation, the scene to confirm the valve status, two district sampling test success rate of $100 \%$.

LORA is mainly used for low-rate communication, it is really with GFSK open communication distance gap rate is lower than $1 \mathrm{kbps}$, in the transmission power of a certain time $(\leqslant 50 \mathrm{~mW})$, spread spectrum table receiving the higher the sensitivity, the longer the communication distance, but at the same time Resulting in lower communication rate, on-site meter reading waiting time will be a corresponding increase, the water company according to the actual needs of the business, choose a good communication rate and transmission distance balance. Pilot spread spectrum table by field test empty environment transmission distance of up to $3.2 \mathrm{~km}$, residential building outside the meter reading signal can cover 34 floors (test environment up to 34 layers), single table communication time up to 10 s.

\section{Conclusion}

Based on the spread spectrum valve control technology, the wireless remote meter reading system uses the spread spectrum LOAR communication as the data transmission technology. The communication method has the advantages of long transmission distance, strong wall resistance, anti-fading, anti-interference and high data confidentiality. In the system, the district management machine to achieve the wireless remote management of the district, data collection and storage, and 
according to the management center system settings, regular copy of the wireless remote table data, through the ABC channel to transfer data to the management center. The wireless remote meter reading system constructed in this way has the advantages of making full use of the existing network, shortening the construction period, reducing the construction cost, equipment installation convenience and simple maintenance. The use of such water meter and its meter reading system can effectively solve the water company charges difficult, arrears and disputes and other issues, so as to comprehensively improve the water management information, digital, automation level.

\section{References}

[1] Wang Wei, Ma Yonggang, Song Chunlin, Ren Weihao. Design and implementation of wireless meter reading system [J]. Technology horizon, 2015 (05)

[2] Liu Ying, Wang Zaiying. Design and implementation of remote wireless meter reading system [J]. Modern Building Electric, 2012 (08)

[3] Xin Zengnian, Ji Chuntao. Intelligent water meter wireless meter reading system design [J]. Science and Technology Innovation Guide, 2008 (36)

[4] Yao Tong Tong, Zhang Jing. Research and development of remote intelligent wireless meter reading system based on mobile communication [J]. Technology Square, 2016 (11)

[5] Li Yongshang. Design of micro - power wireless meter reading system based on ZigBee [J]. Microcomputers and applications, 2016 (16) 